\title{
Haplotypes of $q G L 3$ and their roles in grain size regulation with GS3 alleles in rice
}

\author{
Y.D. Zhang ${ }^{1,2}$, Z. Zhu', Q.Y. Zhao',2, T. Chen', S. Yao', L.H. Zhou', L. Zhao', \\ C.F. Zhao' and C.L. Wang ${ }^{1,2}$ \\ ${ }^{1}$ Institute of Food Crops, Jiangsu Academy of Agricultural Sciences, Nanjing, China \\ ${ }^{2}$ Jiangsu Co-Innovation Center for Modern Production Technology of Grain Crops, \\ Yangzhou University, Yangzhou, China \\ Corresponding author: C.L. Wang \\ E-mail: cailin_wang@sina.com \\ Genet. Mol. Res. 15 (1): gmr.15017587 \\ Received September 3, 2015 \\ Accepted November 5, 2015 \\ Published January 29, 2016 \\ DOI http://dx.doi.org/10.4238/gmr.15017587
}

ABSTRACT. Grain size is an important trait that directly influences rice yield. The $q G L 3$ and GS3 genes are two putative regulators that play a role in grain size determination. A single rare nucleotide substitution $(C \rightarrow A)$ at position 1092 in exon 10 of qGL3 might be responsible for variations in grain size. However, little is known about the haplotype variations of $q G L 3$ and their interactions with GS3 during the regulation of grain length and grain weight. In this study, qGL3 haplotype variations were examined in 61 Indica varieties, and the effects of $q G L 3$ and GS3 on grain trait variation in 110 lines were evaluated. Six qGL3 haplotypes were identified, and qGL32 was a major haplotype in Indica varieties. Moreover, $q G L 3-6$, a reported key single nucleotide polymorphism, was validated. Our results showed that the mutants $q g / 3$ and gs3 (loss-of-function mutation types of $q G L 3$ and GS3, respectively) had significant effects on grain length and grain weight. However, no significant effects associated with differences in the regulation of grain thickness were observed. The genetic effects of $q g / 3$ on grain phenotypes were stronger than those of gs 3 . In addition to increased grain length, $9 g / 3$ had an evident role in grain width increases. In contrast, gs 3 
played an opposite role in grain width regulation. These results provided novel insights into grain size control and the functions of $q g / 3$ and $g s 3$ in rice yield improvement.

Key words: Rice; Haplotype; Grain size; qGL3; GS3

\section{INTRODUCTION}

Rice is one of the most important food crops, and it provides daily diet nutrition for more than half of the global population. Therefore, the stability and increase of rice output is vital to the elimination of hunger and the improvement of quality of life. Grain weight is closely associated with rice yield (Xing et al., 2002). Grain shape, as the major determinant of grain weight, is characterized by a combination of four parameters: grain length, grain width, grain thickness, and filling degree (Xing and Zhang, 2010; Huang et al., 2013; Ikeda et al., 2013; Zuo and Li, 2014). In recent years, several key genes that regulate grain shape were identified (Fan et al., 2006; Shomura et al., 2008; Weng et al., 2008; Li et al., 2011; Zhang et al., 2012; Ishimaru et al., 2013; Song et al., 2007, 2015; Wang et al., 2012, 2015a,b). For instance, qGL3/qGL3.1 is a newly identified QTL associated with grain length, which exhibited the highest logarithm of the odds value and the greatest phenotypic variation compared to other markers, and it also significantly contributed to grain thickness and grain width (Hu et al., 2012; Qi et al., 2012; Zhang et al., 2012). A comparison of functional and nonfunctional sequences revealed a single nucleotide substitution $(C \rightarrow A)$ at position 1092 in exon 10, resulting in the replacement of $A s p(D)$ (a non-functional allele) with Glu (E) (a functional allele) at the 364th amino acid. $q G L 3 / q G L 3.1$ encodes a putative protein phosphatase with a Kelch-like repeat domain (OsPPKL1), which directly dephosphorylates its substrate (CyclinT1,3) to regulate cell division (Qi et al., 2012). Furthermore, genetic and transgenic studies revealed that $q G L 3 / q G L 3.1$ acts as a negative regulator of grain length. The discovery of functional single nucleotide polymorphisms (SNPs) in qGL3 was expected to improve selection for grain weight in rice-breeding programs.

Previous results indicated that GS3 was a negative regulator of grain size, and that it had major effects on grain length and weight as well as minor effects on grain width and thickness (Fan et al., 2006). The complete GS3 protein contains a plant-specific organ size regulation (OSR) domain at the N-terminus, a transmembrane domain, and both a tumor necrosis factor receptor/nerve growth factor receptor family cysteine-rich domain and a von Willebrand factor type $\mathrm{C}$ at the C-terminus (Mao et al., 2010). The OSR domain is the key negative regulator of grain length, whereas the two C-terminus domains have inhibitory effects on OSR function. A GS3 allele, characterized by a $\mathrm{C} \rightarrow \mathrm{A}$ substitution at $165 \mathrm{bp}$, which caused premature termination of the predicted protein, resulted in the complete loss of the functional protein domain. Moreover, this allele is common in rice with long grains (Mao et al., 2010).

Both $q G L 3$ sequence variations and functional relevance have been explored, but the information remains obscure. In addition, the interactions with GS3, a similar gene that regulates grain length, have not been investigated. In this study, $q G L 3$ haplotypes in 61 Indica rice varieties were identified, and the phenotypes of 110 lines with different qGL3 and GS3 genotypes were analyzed. Our study validated the reported SNPs and insertions and deletions (indels) in $q G L 3$, identified additional SNPs in the functional domain, and evaluated the genetic effects of $q G L 3$ in combination with different GS3 genotypes. Our findings provided insight into the control of grain length in rice and will be of value for the improvement of rice grain yield. 


\section{MATERIAL AND METHODS}

\section{Plant material and phenotypic data collection}

A total of 61 Indica varieties were examined, including 49 Oryza sativa L. accessions containing a wide range of Indica (mainly from China), six rice varieties from the International Rice Research Institute (IRRI), and four varieties from other countries. Most varieties were developed through crossbreeding, and some were landraces (Table S1). A total of 110 inbred lines, which were developed from a cross between TD70 (a long grain Japonica variety carrying gs 3 and $9 g / 3$ loss-offunction mutations) and Kasalath (a short grain Indica variety carrying GS3 and qGL3 wild-type alleles) were used to study the combined effects of $q g / 3$ and gs3. In 2013, the 61 varieties and 110 lines were grown within two replicated plots in the experimental field located at the Institute of Food Crops, Jiangsu Academy of Agricultural Sciences, China. Seeds were immediately harvested once they developed to maturation, and they were then wind-dried for 30 days to maintain a water content of approximately $14 \%$. The grain length and grain width of 10 full seeds were measured using vernier calipers, and the mean values were recorded. The total weight of 100 grains from each plant was measured and converted to its 1000-grain weight using an electronic balance (measured to the nearest $0.0001 \mathrm{~g}$ ).

\section{DNA extraction, PCR, and sequencing}

The fresh young leaves of growing plants from each rice variety were harvested, and the cetyl-trimethyl ammonium bromide method was used to extract genomic DNA. The qGL3 sequences were downloaded from the National Center for Biotechnology Information (accession Nos. EF447275 and AK288069). Primer sets, based on the downloaded sequences, were designed to amplify genomic DNA regions containing all $q G L 3$ exons and introns, and Prime STAR HS DNA polymerase (TaKaRa-Bio Inc.) was used. The following primers in Table 1 were used in this study.

Table 1. Primer sequences used for qGL3 gene cloning.
\begin{tabular}{l|l}
\hline Primer name & Sequence \\
\hline qGL3g1R2 & 5'-CCTCCATTCGTATGGCTT-3' \\
\hline qGL3g2F & 5'-TGATGGTAAGCAATTGCTCC-3' \\
\hline qGL3g2R & 5'-TCCTGGGAGTTGTGACAAC-3' \\
\hline qGL3g3F & 5'-CTCATCACTCTGTGATGACATTG-3' \\
\hline qGL3g3F2 & 5'-GAGGTATAGGTAATTGTTGGTTTCC-3' \\
\hline qGL3g3R & 5'-TCTTAACAGGAGCTTTAAGTTGC-3' \\
\hline qGL3g3R2 & 5'-AACTCACGCAATGTCTCCTG-3' \\
\hline qGL3g4F & 5'-CTGCTGCGATACTTATCAGGT-3' \\
\hline qGL3g4R & 5'-TGTTACCGTGTTACGAGGAAGG-3' \\
\hline qGL3g5F & 5'-CACAGGTACTGCAAATAATGC-3' \\
\hline qGL3g5R & 5'-TTCGGAGCCAAACAAGATAT-3' \\
\hline
\end{tabular}

PCR was performed using a standard three-step protocol with 2X PrimeSTAR GC buffer. PCR products were purified using an Axyprep DNA gel extraction kit (Axygen), and were then sequenced on an $\mathrm{ABI} 377$ sequencer using the same primer sets and a BigDye Terminator Cycle sequencing kit. The gene sequences were assembled, and the consensus sequences were used for analyses. Sequences were aligned using CLUSTALX 2.1 (Larkin et al., 2007), and the results were saved in the TASSEL input format. SNPs and indels were detected using TASSEL 4.0 (Bradbury et al., 2007). 


\section{Functional markers for the identification of $q g / 3$ and $g s 3$}

The qGL3-derived cleaved amplified polymorphic sequence (dCAPS) markers resulted in wild-type products that were cleaved into two fragments (21 and $84 \mathrm{bp}$ ) via endonuclease Accl digestion, whereas those associated with the mutants exhibited a single 105-bp fragment. After PCR, the GS3 dCAPS marker products were specifically cleaved using Pstl. The mutant produced a 168-bp fragment, and the wild type produced 110- and 58-bp fragments (Zhang et al., 2015). The following $q G L 3$ and $G S 3$ primer pairs were used: $q G L 3-\mathrm{F}: 5$ '-GATTCTATCTGGTTCAGTGGTAGA3'and qGL3-R: 5'-CCTGCTGCATCTGCACTATAT-3'; GS3-F: 5'-CCCATCTCCCTCGTTTACTT-3' and GS3-R: 5'-GTAAAGACGAGAAGAAATGG-3'. The PCR conditions were as follows: 4 min at $94^{\circ} \mathrm{C}$; 33 cycles at $94^{\circ} \mathrm{C}$ for $30 \mathrm{~s}, 49^{\circ} \mathrm{C}$ for $30 \mathrm{~s}$, and $72^{\circ} \mathrm{C}$ for $1 \mathrm{~min}$; and a final extension at $72^{\circ} \mathrm{C}$ for $10 \mathrm{~min}$.

\section{RESULTS}

\section{qGL3 haplotypes}

qGL3 was recently identified as a regulator of grain length, and analyses of its gene structure, SNPs, and indels indicated that the variations were conserved in exons and introns (Figure 1A). Only eight SNPs and one indel in 8047 bp were newly identified in the 61 rice varieties, despite the presence of 21 exons and 20 introns (Figure 1B). There was only one SNP for every $1 \mathrm{~kb}$, and $87.5 \%$ of the SNPs were found in exon 1 and intron 1 . A total of 54 rice varieties had the same sequences identified as $q G L 3-2$, and only seven accessions shared the other five haplotypes (Figure 1C). A single nucleotide substitution $(C \rightarrow A)$ was identified as the replacement of arginine with glycine. The S_1092 SNP, which was the same as that found in the extra-large grained Japonica N411 variety (Zhang et al., 2012), was identified as qGL3-6 in this study (Figure 1). Five SNPs in the first exon caused two amino acid changes in $q G L 3-3$, including $S \_535$ and S_536 (cysteine to tyrosine) and S_543 (glycine to arginine) (Figure 1). The other two differences in exon 1, the S_530 and S_548 SNPs, did not cause amino acid residue changes. By analyzing the phenotypes of the two varieties with the two amino acid changes, we found that there was no significant phenotypic correlation between the two qGL3-3 SNPs and grain length (Table S1). With the exception of $q G L 3-6$, five other $q G L 3$ haplotypes were short grained, and no significant differences associated with grain length (Figure 2A), width (Figure 2B), or thickness (Figure 2C were observed.

\section{Grain length differences between $q g / 3$ and gs 3 wild types and mutants}

To analyze the phenotypic differences between the qGL3 mutants and wild types, we divided 61 accessions into two groups. According to the sequencing results, one group included lines containing $q G L 3$ mutants, and the other group included lines with $q G L 3$ wild types. Moreover, one group had a single species (TD70) associated with qGL3-6, and another group contained 60 species associated with five $q G L 3$ haplotypes. The average grain length of the wild-type $q G L 3$ grain was $8.75 \mathrm{~mm}$. Regarding grain length, highly significant differences were found between the $q g / 3$ mutants and wild types (Figure $3 \mathrm{~A}$ ). Furthermore, significant differences were detected between grain length in 60 accessions carrying the $q G L 3$ wild type 
and those with the GS3 mutant and wild type (Figure 3B). These accessions were divided into the GS3 mutant and wild-type varieties based on the GS3 dCAPS markers (Figure 4A), which resulted in the numbers 29 and 31 in Table S1, respectively. To compare the grain length of the GS3 wild type, the grain length of the gs 3 mutant was measured at $9.71 \mathrm{~mm}$, which indicated a $1.8 \mathrm{~mm}$ increase (Figure 3B). This result indicated that the gs 3 mutant had strong genetic effects on grain length. TD70 (S1), which carried qGL3-6, was found to contain the gs 3 mutant (Figure 4A and Table S1).

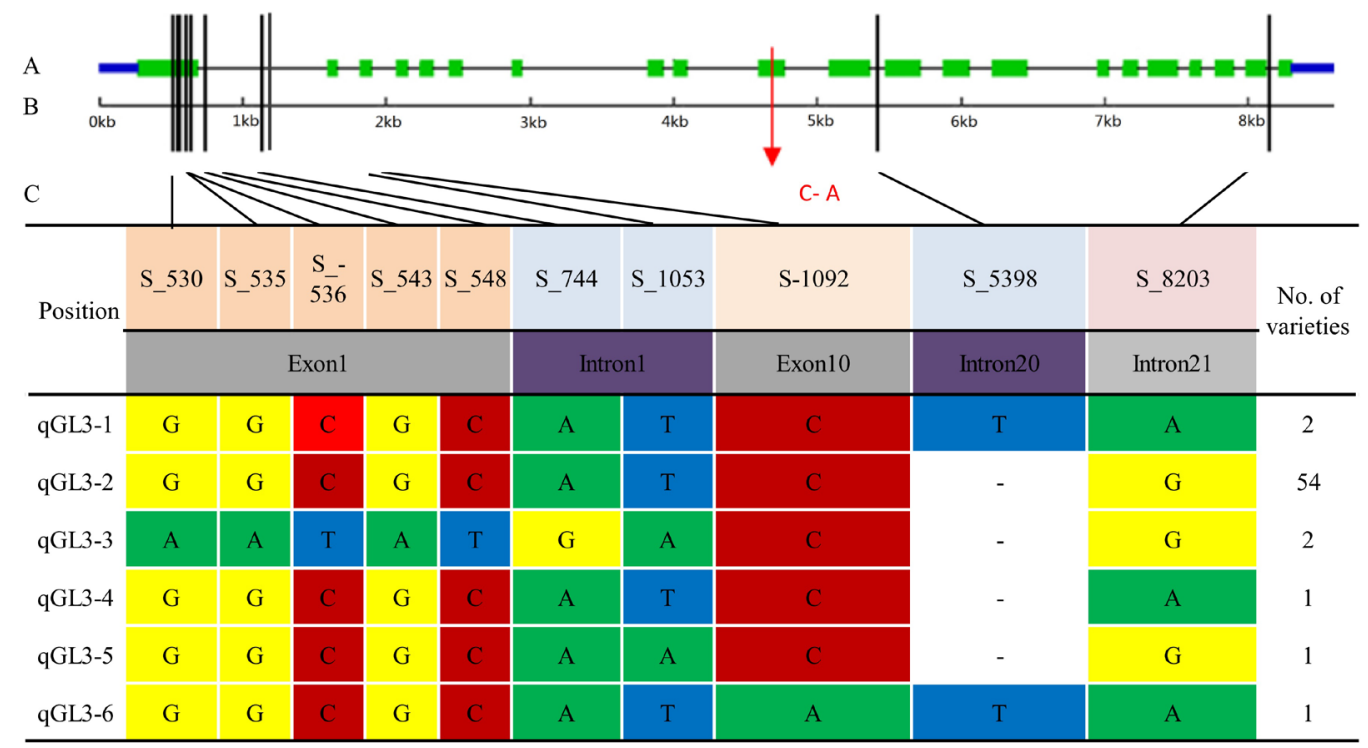

Figure 1. Gene structure, positions of SNPs and indels, and haplotypes of $q G L 3$. A. $q G L 3$ gene structure. Blue boxes indicate upstream/downstream, green boxes indicate exons, and black lines represent introns. B. Gene sequence and positions of SNPs and indels. The vertical lines of the gene structure indicate the positions of polymorphic sites. C. qGL3 haplotypes.
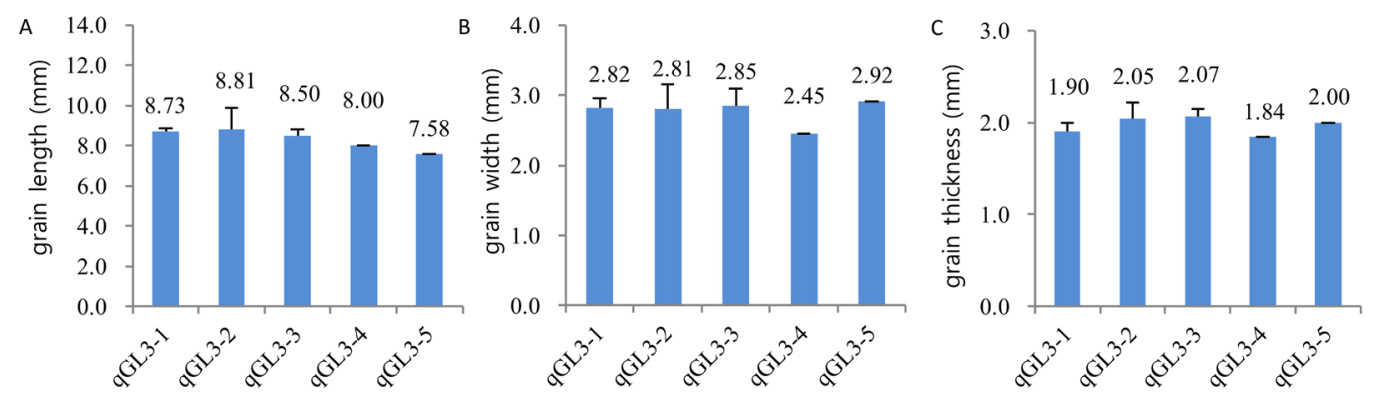

Figure 2. Comparisons of grain phenotypes among varieties carrying different $q G L 3$ haplotypes. A.-C. Grain length, grain width, and grain thickness of different haplotypes, respectively. 

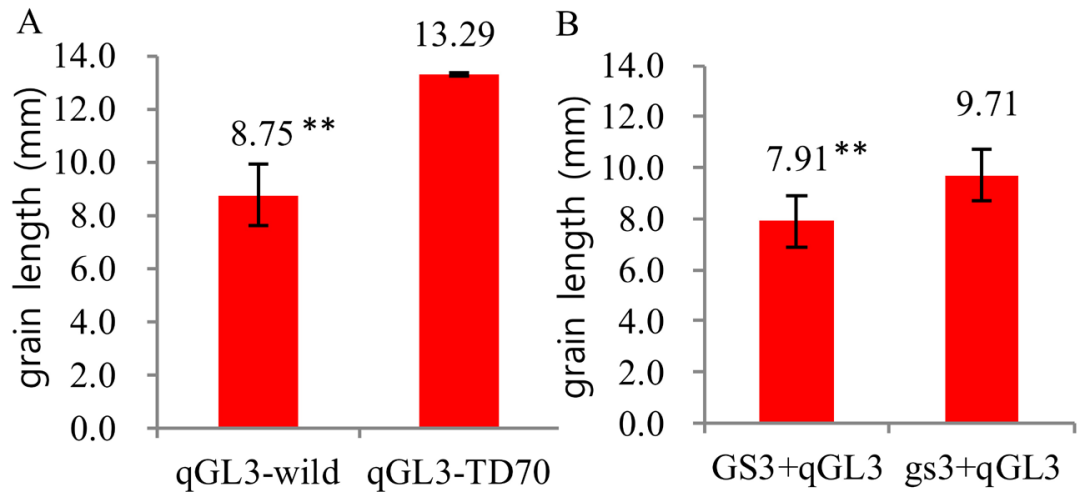

Figure 3. Comparisons of grain length between the mutant and wild-type genotypes of two genes associated with grain length that were found in 61 varieties. A. Grain length of $q G / 3$ and $q g / 3$. B. Grain length of $G S 3+q G L 3$ and gs 3 $+q G L 3 .{ }^{*}$ Significant differences at the 0.01 level.

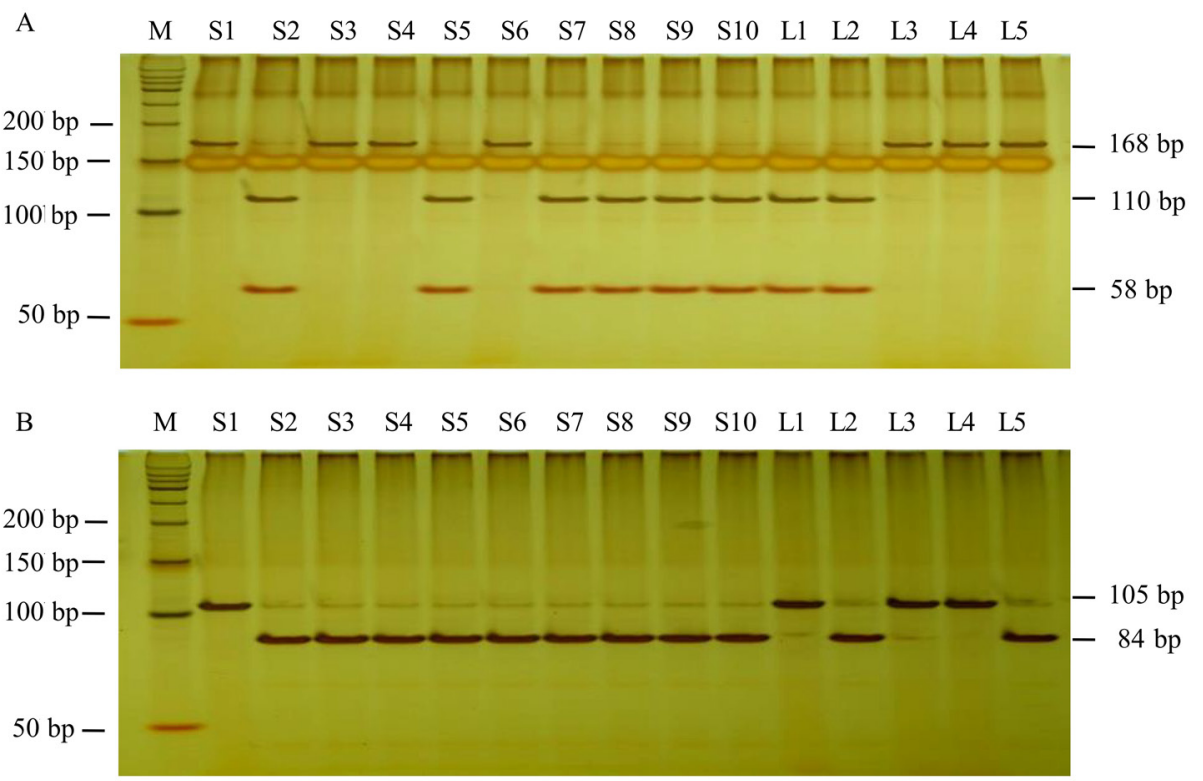

Figure 4. GS3 PCR amplification and enzyme reaction products. A. qGL3. B. Examples of some of the 61 rice variety samples (S1 to S10) and some RILs lines from S1 and S2. Lane M: DNA size marker; lane S1: TD70; lane S2: Kasalath; lanes $S 3$ to $S 10$ : some samples of the 61 rice varieties; lanes $L 1$ to $L 5$ : some RIL lines carrying different GS3 and $q G L 3$ combinations. A 21-bp fragment is not shown in B, because it was too far from the main fragment.

\section{Effects of $q g / 3$ and $g s 3$ combinations}

Of the 61 varieties, a variety containing a qg/3 mutant and a GS3 wild type was not detected, but a variety containing two mutant genes was detected in the 61 varieties (Table S1). To analyze the genetic effects of a $q g / 3$ mutant, we used the effects of two mutant genes to replace those of the $q g / 3$ mutant, and this may have magnified its genetic effect because the influence of $g s 3$ was 
not ignored. We used a dCAPS marker for $q G L 3$ to distinguish the $q G L 3$ genotype, and the results indicated that only TD70 carried the mutant $q g / 3$ in 61 accessions (Figure 4B). To accurately assess the combinatory effects of $q G L 3$ and GS3, 110 lines were established (without selection for $q G L 3$ and GS3) from the $F_{9}$ generation of the hybridization between TD70 (S1) (a variety carrying two mutant genes) and Kasalath (S2) (a variety carrying two wild-type genes), which were selected to detect the genetic effects of $q g / 3$ and gs3 (Figure 4). We divided the 110 lines into the following four classifications based on $q G L 3$ and GS3 molecular markers: 62 lines with $q g / 3$ and gs 3 wild types, 10 lines with one $q g / 3$ mutant, 28 lines with one gs 3 mutant, and 10 lines with $q g / 3$ and gs 3 mutants (which contained 62, 10, 28, and 10 lines, respectively). In order to analyze the combined effects of the two genes, the grain length and grain weight phenotypes were investigated in the following genetic groups: $q g / 3+g s 3, G S 3+q g / 3, g s 3+q G L 3$, and $G S 3+q G L 3$ (Figure 5). The grain length of the $q g / 3+g s 3$ combination was significantly higher than that of the $g s 3+q G L 3$ $(+1.23 \mathrm{~mm})$ and GS3 + qGL3 $(+2.44 \mathrm{~mm})$ combinations (Figure $5 \mathrm{~A})$. The ascending order of the grain width phenotypes was $G S 3+q g / 3, G S 3+q G L 3, q g / 3+g s 3$, and $g s 3+q G L 3$, but a significant difference was not detected (Figure 5B). Furthermore, the same results were found following the analysis of grain thickness (Figure $5 \mathrm{C}$ ). The results of comparisons of the grain weight phenotypes of lines carrying the $G S 3+q G L 3$ genotype combinations ( $g s 3+q G L 3, q g / 3+G S 3$, and $q g / 3+g s 3)$ indicated increases of 4.02, 7.76, and $8.92 \mathrm{~g}$, respectively (Figure 5D). The single mutants, $q g / 3$ $(q g / 3+G S 3)$ and $g s 3(q G L 3+g s 3)$, had significant effects on the regulation of grain length and grain weight, but no significant differences associated with the regulation of grain thickness were detected between the two mutants. The genetic effects of $q g / 3$ on grain traits were greater than that of gs 3 . In addition to the role of increasing grain length, the $q g / 3$ mutant played a specific role in increasing grain width. In contrast, the gs 3 mutant had partially opposite effects on grain width.
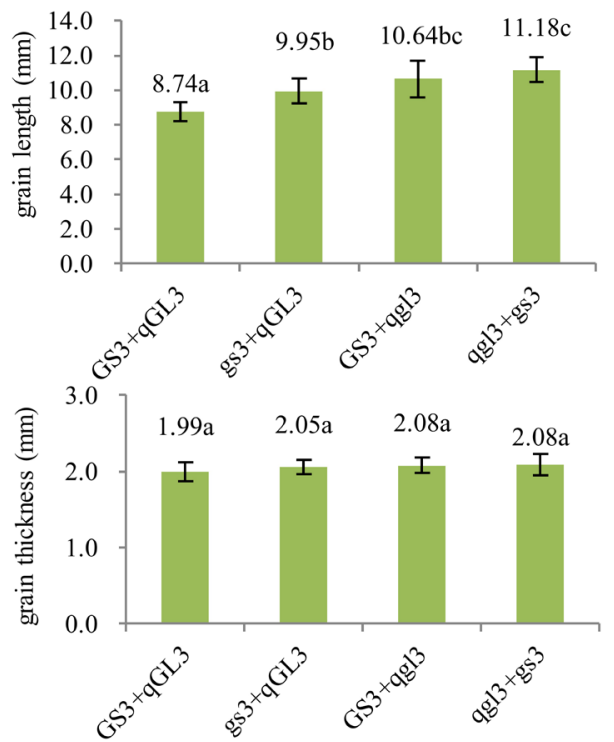

B

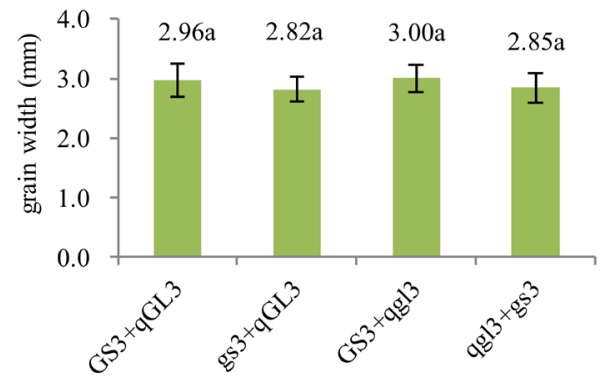

$\mathrm{D}$

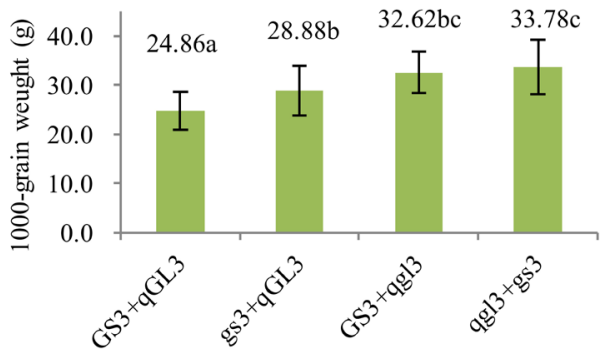

Figure 5. Comparisons of grain traits among different genotype combinations of $q G L 3$ and GS3. A.-D. Grain length, width, thickness, and 1000-grain weight of different combinations, respectively. a, b: ranked using the Duncan test at $P<0.05$. 


\section{DISCUSSION}

\section{Haplotypes of genes associated with grain size}

In recent years, substantial research progress has been made regarding genes associated with grain size, particularly research focused on GS3, qSW5, GW2, GS3, GS5, and TGW6 haplotypes (Weng et al., 2008; Fan et al., 2009; Takano-Kai et al., 2009; Mao et al., 2010; Li et al., 2011; Dixit et al., 2013; Ishimaru et al., 2013; Lu et al., 2013; Xu et al., 2015). Zhang et al. (2012) found that only SNP1 in the $q$ GL3 region in 94 germplasms (the same SNP that was found in TD70) contributed highly to grain length, whereas other polymorphic sites had no significant contributions to grain length. According to the coding sequence, GS3 has at least four different alleles (Mao et al., 2010). Lu et al. (2013) compared the rice grain characters among GS5 and qSW5 genotypes, and the results showed that functional polymorphic sites contributed significantly more to grain traits than other haplotypes. The positive functional alleles of GS3, qSW5/GW5, GS5, and GW8, which are associated with grain size, were common in modern rice varieties (Shomura et al., 2008; Weng et al., 2008; Mao et al., 2010; Li et al., 2011; Yan et al., 2011; Wang et al., 2012). However, the beneficial qGL3 allele was rare (Zhang et al., 2012), and this was similar to GW2 (Song et al., 2007). In this study, six haplotypes were detected in 61 accessions, and only the qGL3-6 allele had great potential for the improvement of grain size. This allele was extremely rare in rice germplasm collections, indicating that grains of this length have not been favored by natural selection or artificial breeding.

\section{Contributions of qGL3 and GS3 to rice breeding}

A major goal of studying genes associated with rice grain size is the improvement of crop yield using the obtained knowledge. A thorough understanding of the function of these genes is essential for applications to breeding. We found that a single nucleotide substitution $(C \rightarrow A)$ at position 1092 in exon 10 resulted in the replacement of Asp (D) (a non-functional allele) with Glu (E) (a functional allele) at the 364th amino acid, which led to increases in grain length and weight. Premature termination of the OSR domain by a single base substitution ( $\mathrm{C} \rightarrow \mathrm{A}$ nonsense mutation in the second exon of GS3) greatly increased grain length, and this mutation is associated with long grain varieties that are widely cultivated around the world. Moreover, this GS3 allele has been highly favored in breeding programs (Mao et al., 2010; Takano-Kai et al., 2011).

Although the location of $q G L 3$ and GS3 genes on chromosome 3 was close, they did not exhibit genetic linkage characteristics (Fan et al., 2006; Zhang et al., 2012). We found that $q G L 3$ can not only be freely combined and separated in the offspring, but it can also be stably integrated with GS3 in the same varieties. Integration of these two genes did not show any inhibitory effects, but the combination of the two mutant genes exhibited stronger effects than either gene alone.

For each individual gene, the effect of $q g / 3$ on the improvement of grain length and grain weight was greater than that of gs3. Therefore, gw8 could shorten grain length when it increases grain width (Wang et al., 2012), but gs3 had the opposite effect. Interestingly, qg/3 could increase both grain length and grain width simultaneously. Because it is widely used in most Indica varieties, the breeding value of the GS3 mutant was confirmed (Takano-Kai et al., 2009; 2011). We believe that when introduced to existing varieties, $9 g / 3$ will play an important role in the improvement of whole rice grain size. 
In summary, qGL3 variations are conserved in exons and introns, and GL3-6 exhibited great potential for grain size improvement. The development of the two markers could accurately distinguish the qGL3 and GS3 mutant and wild-type alleles, which could be used for molecular marker-assisted selection of these two genes. The combination of $q g / 3$ and $g s 3$ generated significant genetic effects, which led to larger grain sizes than observed in either of the parents. The rare $q g / 3$ allele had greater effects on the 1000-grain weight than gs3. These results will provide critical information for breeding programs that seek to modify grain size and increase grain yield.

\section{Conflicts of interest}

The authors declare no conflict of interest.

\section{ACKNOWLEDGMENTS}

Research supported by the National Natural Science Foundation of China (Grant \#31271678), the Jiangsu Agricultural Scientific Self-Innovation Fund (Grant \#CX[14]5107), and the Key Research and Development Program for Modern Agriculture of Jiangsu Province (\#BE2015363-3).

\section{REFERENCES}

Bradbury PJ, Zhang Z, Kroon DE, Casstevens TM, et al. (2007). TASSEL: software for association mapping of complex traits in diverse samples. Bioinformatics 23: 2633-2635.http://dx.doi.org/10.1093/bioinformatics/btm308

Dixit N, Dokku P, Amitha MSV, Parida SK, et al. (2013). Haplotype structure in grain weight gene GW2 and its association with grain characteristics in rice. Euphytica 192: 55-61. http://dx.doi.org/10.1007/s10681-012-0852-4

Fan C, Xing Y, Mao H, Lu T, et al. (2006). GS3, a major QTL for grain length and weight and minor QTL for grain width and thickness in rice, encodes a putative transmembrane protein. Theor. Appl. Genet. 112: 1164-1171. http://dx.doi.org/10.1007/s00122-006-0218-1

Fan C, Yu S, Wang C and Xing Y (2009). A causal C-A mutation in the second exon of GS3 highly associated with rice grain length and validated as a functional marker. Theor. Appl. Genet. 118: 465-472.http://dx.doi.org/10.1007/s00122-008-0913-1

$\mathrm{Hu}$ Z, He H, Zhang S, Sun F, et al. (2012). A Kelch motif-containing serine/threonine protein phosphatase determines the large grain QTL trait in rice. J. Integr. Plant Biol. 54: 979-990.http://dx.doi.org/10.1111/jipb.12008

Huang R, Jiang L, Zheng J, Wang T, et al. (2013). Genetic bases of rice grain shape: so many genes, so little known. Trends Plant Sci. 18: 218-226.http://dx.doi.org/10.1016/j.tplants.2012.11.001

Ikeda M, Miura K, Aya K, Kitano H, et al. (2013). Genes offering the potential for designing yield-related traits in rice. Curr. Opin. Plant Biol. 16: 213-220.http://dx.doi.org/10.1016/j.pbi.2013.02.002

Ishimaru K, Hirotsu N, Madoka Y, Murakami N, et al. (2013). Loss of function of the IAA-glucose hydrolase gene TGW6 enhances rice grain weight and increases yield. Nat. Genet. 45: 707-711.http://dx.doi.org/10.1038/ng.2612

Larkin MA, Blackshields G, Brown NP, Chenna R, et al. (2007). Clustal W and Clustal X version 2.0. Bioinformatics 23: 29472948.http://dx.doi.org/10.1093/bioinformatics/btm404

Li Y, Fan C, Xing Y, Jiang Y, et al. (2011). Natural variation in GS5 plays an important role in regulating grain size and yield in rice. Nat. Genet. 43: 1266-1269.http://dx.doi.org/10.1038/ng.977

Lu L, Shao D, Qiu X, Sun L, et al. (2013). Natural variation and artificial selection in four genes determine grain shape in rice. New Phytol. 200: 1269-1280.http://dx.doi.org/10.1111/nph.12430

Mao H, Sun S, Yao J, Wang C, et al. (2010). Linking differential domain functions of the GS3 protein to natural variation of grain size in rice. Proc. Natl. Acad. Sci. USA 107: 19579-19584.http://dx.doi.org/10.1073/pnas.1014419107

Qi P, Lin YS, Song XJ, Shen JB, et al. (2012). The novel quantitative trait locus GL3.1 controls rice grain size and yield by regulating Cyclin-T1;3. Cell Res. 22: 1666-1680.http://dx.doi.org/10.1038/cr.2012.151

Shomura A, Izawa T, Ebana K, Ebitani T, et al. (2008). Deletion in a gene associated with grain size increased yields during rice domestication. Nat. Genet. 40: 1023-1028.http://dx.doi.org/10.1038/ng.169

Song XJ, Huang W, Shi M, Zhu MZ, et al. (2007). A QTL for rice grain width and weight encodes a previously unknown RING- 
type E3 ubiquitin ligase. Nat. Genet. 39: 623-630.http://dx.doi.org/10.1038/ng2014

Song XJ, Kuroha T, Ayano M, Furuta T, et al. (2015). Rare allele of a previously unidentified histone $\mathrm{H} 4$ acetyltransferase enhances grain weight, yield, and plant biomass in rice. Proc. Natl. Acad. Sci. USA 112: 76-81. http://dx.doi.org/10.1073/pnas.1421127112

Takano-Kai N, Jiang H, Kubo T, Sweeney M, et al. (2009). Evolutionary history of GS3, a gene conferring grain length in rice. Genetics 182: 1323-1334.http://dx.doi.org/10.1534/genetics.109.103002

Takano-Kai N, Doi K and Yoshimura A (2011). GS3 participates in stigma exsertion as well as seed length in rice. Breed. Sci. 61: 244-250. http://dx.doi.org/10.1270/jsbbs.61.244

Wang S, Wu K, Yuan Q, Liu X, et al. (2012). Control of grain size, shape and quality by OsSPL16 in rice. Nat. Genet. 44: $950-$ 954.http://dx.doi.org/10.1038/ng.2327

Wang S, Li S, Liu Q, Wu K, et al. (2015a). The OsSPL16-GW7 regulatory module determines grain shape and simultaneously improves rice yield and grain quality. Nat. Genet. 47: 949-954.http://dx.doi.org/10.1038/ng.3352

Wang Y, Xiong G, Hu J, Jiang L, et al. (2015b). Copy number variation at the GL7 locus contributes to grain size diversity in rice. Nat. Genet. 47: 944-948.http://dx.doi.org/10.1038/ng.3346

Weng J, Gu S, Wan X, Gao H, et al. (2008). Isolation and initial characterization of GW5, a major QTL associated with rice grain width and weight. Cell Res. 18: 1199-1209.http://dx.doi.org/10.1038/cr.2008.307

Xing Y and Zhang Q (2010). Genetic and molecular bases of rice yield. Annu. Rev. Plant Biol. 61: 421-442. http://dx.doi.org/10.1146/annurev-arplant-042809-112209

Xing Z, Tan F, Hua P, Sun L, et al. (2002). Characterization of the main effects, epistatic effects and their environmental interactions of QTLs on the genetic basis of yield traits in rice. Theor. Appl. Genet. 105: 248-257. http://dx.doi.org/10.1007/s00122-002-0952-y

Xu C, Liu Y, Li Y, Xu X, et al. (2015). Differential expression of GS5 regulates grain size in rice. J. Exp. Bot. 66: $2611-2623$. http://dx.doi.org/10.1093/jxb/erv058

Yan S, Zou G, Li S, Wang H, et al. (2011). Seed size is determined by the combinations of the genes controlling different seed characteristics in rice. Theor. Appl. Genet. 123: 1173-1181.http://dx.doi.org/10.1007/s00122-011-1657-x

Zhang X, Wang J, Huang J, Lan H, et al. (2012). Rare allele of OsPPKL1 associated with grain length causes extra-large grain and a significant yield increase in rice. Proc. Natl. Acad. Sci. USA 109: 21534-21539. http://dx.doi.org/10.1073/pnas.1219776110

Zhang YD, Zheng J, Liang ZK, Liang YL, et al. (2015). Verification and evaluation of grain QTLs using RILs from TD70 $x$ Kasalath in rice. Genet. Mol. Res. 14: 14882-14892.

Zuo J and Li J (2014). Molecular genetic dissection of quantitative trait loci regulating rice grain size. Annu. Rev. Genet. 48: 99-118.http://dx.doi.org/10.1146/annurev-genet-120213-092138

\section{Supplementary material}

Table S1. The 61 rice varieties analyzed in this experiment and their grain shape phenotype, genotypes of GS3, and haplotypes of $q G L 3$.

\section{http://www.geneticsmr.com/year2016/vol15-1/pdf/gmr7587 supplementary.pdf}

